\title{
Patient considerations in the management of multiple sclerosis: development and clinical utility of oral agents
}

This article was published in the following Dove Press journal:

Patient Preference and Adherence

| March 201 |

Number of times this article has been viewed

\author{
Nathalie Girouard \\ Nanda Soucy \\ MS Clinic, Ottawa Hospital, Ottawa, \\ Ontario, Canada
}

\begin{abstract}
Multiple sclerosis (MS) is one of the most frequently occurring disabling neurological disorders among young adults in Canada. It is a chronic inflammatory disorder of the central nervous system (CNS) that is thought to be immune mediated in nature. An estimated 55,000-75,000 Canadians suffer from this debilitating disease. Starting in the mid-1990s, we witnessed the beginning of a new era in the treatment of MS. Treatments finally became available to help modify the course of the disease. Early initiation of treatment soon after diagnosis has become the expectation in many MS clinics, warranting many decisions to be made by the patient with the assistance of their health care team. Currently, there are two categories of disease-modifying therapies (DMTs) available: immunomodulatory and immunosuppressant agents. Although disease-modifying therapies are not a cure and are only moderately effective, they offer a possible slowing of any progression that may occur over time, a decrease in relapse activity, and a decrease in the amount of new lesions developing in the CNS found on magnetic resonance imaging. Not only have these agents been partly effective but up to now they have only been available parenterally, which has many limitations, including a major factor in determining the best outcome for the treatment: adherence. Four new DMTs will likely become available to Canadians over the next five years. Fingolimod, cladribine, teriflunomide, and laquinimod are likely to be marketed as the first oral DMTs in Canada. The US Food and Drug Administration approved fingolimod in September 2010 as a first-line therapy for relapsing forms of MS. Dalfampridine is also available in the US as an agent able to improve walking. Even if these agents present with higher efficacy and a promising safety and tolerability profile, thus possibly demonstrating better adherence, it will be imperative for the health care professionals to focus on monitoring and supporting the patient to ensure reliable reporting of side effects and to improve overall adherence. In the near future, more treatments will become available to the MS population, and choices will become even more complex so that ongoing support, open communication, and education are required to tame any uncertainties about decisions made regarding treatments.
\end{abstract}

Keywords: adherence, multiple sclerosis, treatment, decisions

\section{Educated decision making}

Every day a person is faced with making multiple choices that define them as individuals, often not realizing that decisions are being made by them and for them on a continuous basis. In the multiple sclerosis (MS) world, when it comes to treatments, today's patients are faced with multiple decisions on choosing the best treatment for them, often leaving them overwhelmed. They and their health care professional team are faced with challenges surrounding the management of their MS. Together, it is anticipated that the "right" decision will be made. One of the drawbacks of the existing
Correspondence: Nanda Soucy 50I Smyth Road Box 606, Ottawa, Ontario, KIH 8L6, Canada

Tel +l 6137378532

Fax + $613739663 \mid$

Email nsoucy@toh.on.ca 
and newer therapies for MS is the lack of any reliable markers for determining which agent will work best for a given person, ${ }^{1}$ so often the overall treatment decision is made by trial and error. There is an art to decision making that most individuals are not aware of; most may not consider what may be the motivating or influencing factors that affect their choice, and they are more complex than one may think.

First, patients need up-to-date evidence-based information and decision support systems in order to make informed decisions together with physicians based on their personal preferences. ${ }^{2}$ Second, there are a host of new agents that are becoming available to treat MS, thus making the choice of treatment that much more challenging. Third, a patient's individual attitude toward risk often plays a key role in what he or she will choose. ${ }^{1}$

Together, the health care professional and the patient (along with their family and friends) will be exploring many questions. Should I go on treatment or wait? What is important to me where my health is concerned? What are my priorities? Should I enter a clinical trial or go with standard therapy? Which treatment should I choose? What are the side effects? How will the treatment affect me? How much does it cost? What are the short- and long-term consequences of taking a particular treatment?

Each patient presents themselves in the clinic with the need for more information. ${ }^{1}$ It is important for the health care professional to view each patient as unique. There is a commonality among them: they all have MS, but they are individuals with different needs, wants, and desires. In conversation with patients, it is important for the nurse and/or physician to assess what type of individual they are facing ("internal" or "external"), and an adjustment to the way they communicate their messages is required in order for them to be most impactful for a variety of patients. ${ }^{3}$ An internal person will make a decision based on their own internal criteria and rely on what they know, so may not gather enough information. They rely on their own intuitive judgment to make decisions and they focus on what they want. ${ }^{3}$ An external person relies on others to help them decide what to do. The decision is based on outside criteria and the focus is on others. They are often easily influenced. ${ }^{3}$ The time that is taken with the patient when they learn of their diagnosis and when treatment is considered is crucial and could easily determine the future plan of the patient with MS based on their choices. One-on-one conversation will reveal whether someone is willing to take greater risks or someone who is more comfortable waiting to see what will happen over time, thus establishing what the next step for follow-up will be.
The concept of early diagnosis and early treatment adds to the hastening of suggested treatment decision making. At the same time, if a person is rushed into making a decision they are not ready to make, especially when there are many options to choose from, it might leave them with a feeling of regret and of having an unpleasant experience, ${ }^{4}$ or leave them questioning whether they made the right choice. Educating patients about the pros and cons of available MS therapies requires time, knowledge, appropriate teaching tools, and follow-up. ${ }^{1}$ The need for the health care professional to involve the patient in the treatment decision making process is imperative in beginning to build a trusting relationship. Every choice, whether life altering or not, has the potential to leave us feeling anxious or regretful. Anxiety is an emotion of the future that you can only experience in the now by imagining something that has not happened yet turning out in the way you do not want it to. ${ }^{3}$ The unknown can be frightening for many who are faced with making a decision; some find it impossible to accept they need to make a change in their life. We have the power to reduce the exhausting effects of choice, not by expanding patients' options but by delegating parts of a decision to others or by limiting them in ways that positively affect the choosing process. ${ }^{4}$ One example of these strategies is consulting experts when one is too emotionally tied to the situation to make a sound judgment. ${ }^{4}$ As professionals, we can encourage patients to see that with change comes growth, learning, and new discoveries. Patients' well-being for maintaining independence as long as possible is always in mind.

Starting an MS treatment or switching medications is one proactive way the patient can gain some control over their future. Much hope is riding on the new wave of medications to help manage the disease in people who are not well controlled on the standard therapies. ${ }^{1}$ Expectations are high that the oral therapies will be an easy alternative to injectable therapies. What people are slowly realizing is that increased safety risk may be the biggest challenge of all with more efficacious medication. The question "Should I start a medication?" is slowly becoming "What medication should I start?" and "What am I willing to risk?". Many patients will assume that because the research demonstrates moderate reduction in relapses or magnetic resonance imaging (MRI) activity, which are overall positive outcomes, their individual journey with MS will reflect just that. Realistic expectations from the beginning are crucial in increasing the likelihood of adherence maintenance. Education on the disease process, the natural history without treatment, and the effect of treatment on the disease must be articulated. ${ }^{5} \mathrm{~A}$ balanced approach in 
educating patients on both the advantages and disadvantages of the various drug products also serves to promote important input by our patients, which can ultimately lead to an appropriate choice of therapy and a greater likelihood of compliance and tolerability. ${ }^{5}$ Although many patients may prefer an oral MS drug, the ultimate choice of therapy will be a shared patient-physician-nurse decision based on a multitude of factors. ${ }^{6}$ With the emergence of new targeted therapies, better efficacy may be achieved. Oral route for administration plus less frequent administration may improve adherence. Long-term safety data still remain an unknown. Close monitoring of effects is warranted and may pose as a challenge in some clinic settings. Ultimately, MS patients can achieve maximum benefits from these agents if they are reasonably well tolerated and used with consistency. ${ }^{5}$

\section{Emerging oral agents for multiple sclerosis}

Many therapies are likely to be approved for the Canadian market over the next 5 years for MS, the majority of them in the long-awaited oral form, eg, fingolimod, cladribine, and laquinimod, as well as dalfampridine used as a walking enhancer.

\section{Fingolimod}

Fingolimod is a sphingosine 1-phosphate receptor modulator that binds to the receptor on the circulating lymphocytes and therefore interferes with cell migration from the lymph nodes. ${ }^{7}$ It reduces T-cell infiltration in the central nervous system (CNS). ${ }^{8}$

A placebo-controlled Phase II study by Kappos et $\mathrm{al}^{9}$ randomized 281 patients to receive $1.25 \mathrm{mg} /$ day, $5 \mathrm{mg} /$ day, or placebo over a period of 6 months. A blinded 6-month extension study followed, where placebo patients were then randomized to receive either dose of fingolimod. The treated patients showed a significantly reduced number of active inflammatory lesions and significantly better annualized relapse rates (ARRs) than placebo. Although the outcomes were similar for both treatment arms, the adverse events (AEs) were significantly more frequent for the $5 \mathrm{mg} /$ day patients than for those on placebo or lower-dose fingolimod. ${ }^{9}$ The most commonly reported side effects were nasopharyingitis and dyspnea (more common in the $5 \mathrm{mg}$ /day group), headache, diarrhea, and nausea. A decrease in heart rate by a median of 13.8 beats per minute (BPM) was reported for the $1.25 \mathrm{mg} /$ day group and 16.6 BPM for the patients on $5 \mathrm{mg}$ /day. ${ }^{9}$ In light of the increased risk of AEs in the higher-dose group, a different dosing schedule was arranged for subsequent trials.
The FREEDOMS (FTY720 Research Evaluating Effects of Daily Oral Therapy in Multiple Sclerosis) trial, a Phase III, 24-month, placebo-controlled trial extending over 24 months, found similar results. The ARR and MRI variables (the number of Gd(gadolinium)+-enhancing lesions and absence at 24 months) were significantly better for both the $0.5 \mathrm{mg}$ /day and $1.25 \mathrm{mg} /$ day arms. ${ }^{8}$ Both arms also showed a significant positive effect on the cumulative probability of disability progression. ${ }^{8}$

In a head-to-head trial, the safety and efficacy of fingolimod were compared with interferon $\beta$-1a (IFN $\beta$-1a) intramuscularly. The 12-month TRANSFORMS (Trial Assessing injectable interferon vS FTY720 Oral in RrMS) showed significantly better MRI outcomes (number of new $\mathrm{T} 2$ or enlarged $\mathrm{T} 2$ lesions or Gd+- enhancing lesions on T1) versus the IFN $\beta-1 \mathrm{a} .{ }^{10}$ The reports of AEs were consistent with prior studies. Key safety observations highlighted by these studies were bradycardia/arrhythmias, macular edema, infections, and possibly cancer. There is no established relationship between fingolimod and causation of cancer, ${ }^{10}$ but close monitoring of the patient is a must by all health care professionals. $^{11}$

\section{Cladribine}

Cladribine is a synthetic purine nucleoside analog. It selectively reduces the number of dividing and nondividing lymphocytes. ${ }^{12}$ It is a therapy with a short-course dosing. It has a well-known safety profile derived from more than 15 years of use of the parenteral formulation in both the oncology field and MS. ${ }^{13}$

Cladribine has many properties that are relevant to MS treatment. It shows sustained reduction in lymphocyte subtypes (CD4+ T cells, CD8+ T cells, and B cells), ${ }^{14}$ has relatively transient effects on other immune cells such as neutrophils and monocytes, ${ }^{15}$ reduces levels of proinflammatory chemokines, ${ }^{16}$ and has the possibility of crossing the blood-brain barrier. ${ }^{17}$

Cladribine was first researched in MS using parenteral administration for both relapsing and progressive patients. These studies demonstrated significant positive outcomes for MRI variables ${ }^{15,18,19}$ and reduction and severity of relapses in the relapsing patients. CLARITY (CLAdRIbine Tablets treating multiple sclerosis orally) emerged as a trial using the developed oral formulation of cladribine. This Phase III trial compared two different dosages $(3.5 \mathrm{mg}$ and $5.35 \mathrm{mg} / \mathrm{kg}$ over two to four short courses annually) against placebo over a period of 96 weeks. The group with patients who had been randomized to either treatment showed significantly 
reduced ARRs, more relapse-free patients, fewer Gd+enhancing lesions, fewer active T2 lesions, and fewer combined lesions.

As expected, lymphopenia was the most frequent $\mathrm{AE}$ reported; infection rates were similar across all groups. No case of herpes zoster was reported in the placebo arm versus 8 (3.5 mg/day) and 12 (5.75 mg/day). Four cases of cancer were reported for the treatment group, but it is unclear whether cladribine had a causal effect.

Two ongoing studies, ONWARD (Oral Cladribine Added on to Interferon Beta-1a in Patients With Active Relapsing Disease) and ORACLE (Oral Cladribine in Early MS), will respectively study the safety and tolerability of cladribine as an add-on to IFN $\beta$-1a subcutaneously and as an early treatment for patients with clinically isolated syndrome in delaying conversion to clinically definite MS.

\section{Teriflunomide}

Teriflunomide is an active leflunomide metabolite that is being extensively investigated for the treatment of MS. It produces a selective depletion of B- and T-cell lymphocytes by inhibiting a key enzyme involved in pyrimidine synthesis. $^{20}$

A Phase II study comparing placebo with $7 \mathrm{mg}$ and $14 \mathrm{mg}$ daily of teriflunomide showed a significant reduction in MRI activity and improved clinical outcomes and was well tolerated with AEs similar between all groups. ${ }^{21}$ An 8-year extension showed continued effect with no new safety concerns. ${ }^{22}$

Combination therapy of teriflunomide added on to glatiramer acetate or IFN $\beta$ is also showing promising results. A recently completed Phase III study reported a significant reduction in relapse rate, disability progression (higher dose), and MRI activity compared with placebo while maintaining a good safety profile. ${ }^{23}$ An extension for this trial is ongoing.

\section{Laquinimod}

Laquinimod, an oral immunomodulator, was developed following an early halting of a Phase III trial involving its predecessor, linomide, due to serious side effects. Linomide has shown promising results with the animal model of MS and in subsequent trials involving MS patients. Laquinimod offers promising results and a better safety profile.

The first Phase II trial showed significant results for the $0.3 \mathrm{mg} /$ day dosage (no significance for the $0.1 \mathrm{mg} /$ day arm) on cumulative number of Gd+-enhancing lesions. ${ }^{24}$ Learning from these dose-dependent results, a Phase IIb trial was developed to compare the possibility of better results while maintaining a favorable safety profile using a higher dose ( $0.6 \mathrm{mg} /$ day). A significant reduction in cumulative number of $\mathrm{Gd}+$-enhancing lesions and other MRI outcomes was found for the $0.6 \mathrm{mg}$ /day group. However, it did not reproduce the significance previously found for the lower-dose group, which could partly be explained by a borderline significance in the first trial. ${ }^{25}$ An extension to this trial shows ongoing significant reduction of MRI activity favoring the $0.6 \mathrm{mg} /$ day dosage. ${ }^{26}$ Two ongoing Phase III trials (ALLEGRO [Assessment of Oral Laquinimod in Preventing Progression of MS] and BRAVO [Benefit-Risk Assessment of Avonex ${ }^{\circledR}$ and Laquinimod]) will provide further data and hopefully show ongoing favorable safety data. Elevation in liver enzymes was the main reported AE for the completed studies.

\section{Dalfampridine}

Dalfampridine is a potassium channel blocker approved by the US Food and Drug Administration for use as a walking enhancer in $\mathrm{MS}^{27}$ It has no indication as an immunomodulator, as it does not reduce relapses or slow progression. Two recent Phase III trials have shown consistent improvement in walking from $35 \%$ to $42.9 \%$ using the Timed 25 -foot Walk test. ${ }^{28,29}$ Increased seizure risk remains the main concern however, good efficacy of the medication and the pros outweighing the cons will likely determine adherence to the treatment.

Do the oral medications have the potential to replace the current injectable therapy or will there remain a place for both oral and injectable treatment in the MS world?

The availability of a number of effective treatment options for patients with relapsing MS offers the patient perspective of many years of well-controlled disease and consequently of more independence and better quality of life..$^{30}$ However, open, educated discussions with the patients regarding efficacy and to clarify expectations are required to maximize adherence capabilities. One possible problem with oral medications is that they may be inaccurately perceived as less effective than injections, which may lead to poor adherence. ${ }^{6}$ Patients who are newly diagnosed with MS may prefer oral agents when they become available. In patients currently receiving therapy, clinicians may be more reluctant to alter successful therapy. Those patients with comorbidities may also have limited choice for starting on oral therapy or changing treatment due to an increased risk of adverse events such as immunosuppression or severe bradycardia. The primary concern is that the treatment should have a long-term, sustained efficacy and a good tolerability 
to encourage patient adherence. ${ }^{31}$ Because most patients with relapsing-remitting MS are young women, it is important to consider the oral agents' potential effects on fertility. ${ }^{31}$ The choice is not obvious, however, when planning the lifelong therapy needed for a chronic debilitating disease. ${ }^{31}$ The need for long-term therapy can present a significant challenge for the MS nurse and an understandable source of concern for the person with MS..$^{32}$ Educating the patient and supporting them to stay on treatment, not just starting it, is a commitment between the health care professional and the patient that is necessary in maintaining adherence.

\section{Adherence}

Patients' nonadherence to medication remains an important concern for treating physicians in all areas of practice. All approved DMTs for MS presently prescribed in Canada are injectables aimed at reducing neurological activity and preventing further disability. First-line treatment options presently available subcutaneously are IFN $\beta$-1a three times a week, IFN $\beta$-1b every second day, and glatiramer acetate given daily, and another version of IFN $\beta$-1a is administered intramuscularly once per week. All of these therapies are given parentally and although they have a good long-term safety profile have been associated with systematic side effects and injection-related site reactions, which can have a big impact on patients' commitment to maintaining the proper administration schedule, therefore affecting outcome. Second-line therapies include an appealing monthly infused natalizumab that has been linked to a risk of the development of progressive multifocal leukoencephalopathy, a potentially fatal opportunistic brain disease, and mitoxantrone, which is approved for secondary progressive MS patients but linked to potential cardiotoxicity. ${ }^{33}$

A recent observational study conducted online with nearly 800 patients showed that the main reason for noncompliance was that patients just forgot to take their medication. ${ }^{34}$ Often, as a health care professional, we may find ourselves asking our patient "What is it that makes you forget to take your medication?" or telling them "Make sure you find ways to not forget to take your medication". McGarvey, from Solutions in Mind, suggests a different approach that health care professionals can take when communicating with our patients. ${ }^{3} \mathrm{He}$ suggests that we focus our conversation on the positive aspect and say "What do you think will help you remember to take your medication?", "How do you see yourself remembering to take your medication?", or "Here are some tips to help you remember". ${ }^{3}$ One will find that adherence will improve if the negative aspect of the conversation is removed.
Other factors that may influence adherence to DMTs that need consideration are the patient's health status, quality of life, disability level, employment, comorbidities, insurance status, prescription copay, and duration of disease. ${ }^{35}$ One study found higher adherence and improved disease when the patient had better mental health and health status overall, pain interference scores, employment, and lower EDSS (Expanded Disabilty Status Scores). ${ }^{35}$ Reasons for nonadherence are numerous, including memory deficit, poor instructions or patient-physician relationship, medical regimen, adverse effects of medication, patient disagreement with the need for treatment, cost, and poor social support. ${ }^{35}$

Although present and emerging therapies are preventive and not curative, long-term adherence will ensure the best possible outcome for the patients, as adherence to treatment has often been the most influential factor when talking about the efficacy of a medication. It is a common belief that adherence will dramatically improve by having DMTs available in the oral form. However, recent reviews of treatment and adherence revealed ongoing concerns with oral treatment in chronic conditions such as cancer, epilepsy, diabetes, and ulcerative colitis and for other preventive measures in which good adherence would obtain near 100\% efficacy (oral contraceptives).

Even if a study conducted by Turner et $\mathrm{al}^{36}$ identified injection anxiety as a focus point in improving patients' adherence, the observational study reported that injection anxiety was accounting for only $3 \%$ of failure to follow prescribed regimens.

Although the oral agents do eliminate the injection-related barriers, according to Treadaway et al, ${ }^{34}$ we are still left with two-thirds of patients expressing multiple factors for their nonadherence. With the oral therapies, patients may not feel as much of the systemic side effects (such as flu-like symptoms) one would experience with interferons, thus will hopefully be more adherent on the oral agents. However, with more potent therapies, the health care team will have to shift their focus of adherence to crucial monitoring and follow-up involved with these novel agents.

The perceived or real lack of efficacy will remain a challenging area, as the new oral agents are still only preventive. It is well documented that patients who have an overly optimistic view regarding efficacy are likely to discontinue therapy. ${ }^{37}$ They may become discouraged if a relapse occurs and may perceive this as a failure of treatment and therefore be less adherent to it, causing a greater shift away from optimal response. Whether this lack of efficacy is real or perceived, it remains an important factor affecting adherence. In a recent study assessing long-term adherence, it was 
found that over a mean duration of 4.2 years, $46 \%$ discontinued therapy; $26 \%$ of all patients discontinued because of a perceived lack of efficacy. Just over $95 \%$ of these patients switched to a different agent. ${ }^{38}$ It will be increasingly important for the counseling professional to inform patients that the oral agents remain a preventive measure and not a cure.

It is imperative that an accessible and reliable established support system is in place in order to encourage adherence and maintain open communication between the patient and health care professional. In addition to the MS clinic nurse specialist, industry-run support call center programs are another means of providing added support to the patient who is considering DMT, who is starting on or switching types of DMT, or who has been on DMT for an established amount of time. Through our experience, it is known that adherence can be negatively affected if support is poor or if the patient feels alone on their journey or that no one is listening to their concerns. The patient needs to feel confident that those they seek advice from are competent, knowledgeable, and empathetic. Such traits may be discovered by the patient during the initial interview with the physician or nurse when considering DMT. The choice of words and techniques used when getting to know the patient and introducing long-term medication as the treatment for their MS can easily influence whether a medication will be chosen and adhered to. Shea, ${ }^{39}$ who speaks about improving medication adherence in general, hopes the term "medication compliance or adherence" will be replaced by "medication interest". It is important for the physician or nurse to find out what the medication means to the individual, as their interest in taking it will be stronger the more there is a meaningful attachment to the outcome of taking it. This can be a major determinant of long-term medication adherence or "interest".

From our experience, we know that when teaching a patient about MS and introducing the different treatments to a patient, something to be mindful of is that people learn in many different ways, so it is important that a variety of teaching methods is available in order for the physician or nurse to adapt based on the needs (and "interest") of the patient. Having tools that are visual, such as posters, flip charts, or models, that can be interactive will be effective for some patients and they will be able to relate to what is being taught to them. Having different types of literature available, such as brochures, pamphlets, clinical studies, articles, and websites, is helpful so that the patient can take something home with them and review the information in their own time. Videos showing the mechanism of action of the medication, people injecting themselves, or patient testimonies may speak to some patients considering DMT. As the relationship between the health care professional and the patient develops, a better understanding of each other's needs will be discovered. MS patients may be more receptive to the fact that a treatment is being recommended to them because their health and maintenance of their independence is the focus, not simply because a physician wants to prescribe drugs and tell them what to do. Shea shares many techniques that the health care professional could choose to use during the interview that will promote an interest of the patient in what is recommended by the physician and nurse. Hopefully, with a combined effort, "the patient will see the medication as a relief from their disease". Here are some tips on interview techniques that we have modified to relate to a person who has MS. Shea hopes the health care professional will pick and choose from the list when interviewing a patient. He states that the health care professional should choose the techniques that appeal to them, because we are all different and must develop our own styles.

1. Learn the patient's belief set. Do they see themselves as having something wrong with them? It is difficult to do so if their symptoms have cleared and they feel "normal" again, so the role of the health care professional would be to help the patient "see" their illness. Are they motivated to take medication for their disease? Do they see that the pros of taking medication outweigh the cons? Often, those who adhere to the regimen know of someone with the same disease and see the results of not taking the medication. In MS, it is difficult to see the immediate effects of taking medication, as it is seen over time with the reduction of relapses or MRI activity. Patients may lose their motivation to stay on treatment because of this.

2. One question Shea suggests could be asked is "Is there anything that your MS is keeping you from doing that you really wish you could do again?". He calls it an "inquiry into lost dreams" technique. The health care professional would then point out the benefits over time of being on a DMT.

3. Become familiar with the patient's unique family history and cultural beliefs in order to discover whether there are any other motivators for taking the medication. If someone does not believe in medication for themselves, perhaps they will take it so they can remain healthy for the sake of their family members, or they may have a family member whose disease worsened because they were not on medication. A visual reminder may also be suggested where the patient takes a photo of their loved one and keeps it with their medication box or autoinjector.

4. Give the patient a sense of collaboration in the decision process, eg, "Together we want to find a medicine that you 
are genuinely 'interested' in taking because it will maintain your independence for a longer time, it will reduce the risk of you having more frequent relapses ..."

5. Learn the patient's opinion and attitude toward taking medication in general. They may have had a negative experience in the past, which can influence their opinion of the medication being suggested in the present and shape their interest and reliability to remain on the dosing schedule. One of the most important questions to ask in an engaging manner is "Do you take your medication as prescribed?". If they do not, then it is the health care professional's need to uncover the reasons why. If they say they are "sensitive to medication", for example, whether they are experiencing regular side effects or there truly are sensitive issues this will need to be explored on an individual basis.

According to Shea, follow-up appointments require just as much attention when speaking to the patient in order to encourage maintenance of the chosen medication. The focus will then be on side effects, effectiveness of the medication, missed doses, continued affordability, and any other pros and cons of their medication that arise. ${ }^{39}$

\section{Conclusion}

Over the next 5 years, several novel therapies for MS are likely to become available for the physician treating patients with MS. ${ }^{40}$ The real challenge lies in the shared decision-making process between health care professionals and patients and in establishing a caring relationship. It is important to establish trusting relationships and effective communication, to actively listen to each other's concerns from both parties' perspectives, and to determine what the barriers may be or that may arise when one chooses to start on therapy as well as to adhere to the regimen. The first few months after starting an MS treatment are crucial in determining whether a patient will continue with therapy or abandon it. Their response within 3-6 months to initial therapy has a big impact on adherence and is usually related to side effects. ${ }^{41}$ Longer duration of adherence may be influenced by doubts that the patient may have related to the effectiveness of therapy. ${ }^{35}$ A continuous re-evaluation of each individual's situation is necessary in order to remain in touch with the experience of the patient. This becomes a team approach right from the beginning after the diagnosis of MS is confirmed and continues with each follow-up visit.

\section{Disclosure}

The authors report no conflicts of interest in this work.

\section{References}

1. Modifying the Immune System in MS: What We Know, What We're Learning. Counseling Points. 2010;6(3).

2. Heesen C, Solari A, Giordano A, et al. Decisions on multiple sclerosis immunotherapy: new treatment complexities urge patient engagement. J Neurol Sci. 2010, doi:10.1016/j.jns.2010.09.012.

3. McGarvey S. Communication Strategies for Interactions with MS Patients. Solutions in Mind, Optimizing Performance. Proceedings of the 1st MS Expert Nurse Summit conference; November 20-21, 2010; Vienna, Austria. Mississauga, 2010.

4. Iyengar S. The Art of Choosing. New York: Hachette Book Group; 2010.

5. Frohman E, Phillips T, Kokel K, et al. Disease-modifying therapy in multiple sclerosis: strategies for optimizing management. The Neurologist. 2002;8:227-236.

6. Lipsy RJ. Will the newer oral MS agents be welcomed by managed care organizations? Am J Manag Care. 2010;16:S227-S233.

7. Brinkmann V. FTY720 (fingolimod) in multiple sclerosis: therapeutic effects in the immune and the central nervous system. Br J Pharmacol. 2009;158(5):1173-1182.

8. Kappos L, Radue EW, O'Connor P, et al. A placebo-controlled trial of oral fingolimod in relapsing multiple sclerosis. N Engl J Med. 2010; 362(5):387-401

9. Kappos L, Antel J, Comi G, et al. Oral fingolimod (FTY720) for relapsing multiple sclerosis. $N$ Engl J Med. 2006;355(11): 1124-1140.

10. Cohen JA, Barkhof F, Comi G, et al. Oral fingolimod or intramuscular interferon for relapsing multiple sclerosis. $N$ Engl J Med. 2010; 362(5):402-415.

11. US Food and Drug Administration. NDA approval letter. September 21, 2010. GYLENIA, NDA no. 022527. http://www.accessdata.fda.gov/ drugsatfda_docs/label/2010/022527s0001bl.pdf. Accessed December 10, 2010.

12. Carson DA, Wasson DB, Taetle R, et al. Specific toxicity of 2-chlorodeoxyadenosine toward resting and proliferating human lymphocytes. Blood. 1983;62:737-743.

13. Gasperini C, Ruggieri S, Pozzilli C. Emerging oral treatments in multiple sclerosis: clinical utility of cladribine tablets. Ther Clin Risk Manag. 2010;6:391-399.

14. Rieckmann P, Comi G, Cook S, et al. Effects of cladribine tablets on peripheral lymphocyte subtypes implicated in multiple sclerosis immunopathegenesis: surface marker analysis for a subset of patients from the 96 week, Phase III, double-blind, placebo-controlled CLARITY study. Presented at the annual Congress of European Committee for Treatment and Research in Multiple Sclerosis (ECTRIMS), Dusseldorf, Germany, September 9-12, 2009. Mult Scler. 2009;15: S248-S249.

15. Rice GP, Filippi M, Comi G. Cladribine and progressive MS: clinical and MRI outcomes of a multicenter controlled trial. Cladribine MRI Study Group. Neurology. 2000;54:1145-1155.

16. Bartosik-Psujek H, Belniak E, Mitosek-Szewczyk K, et al. Interleukin-8 and RANTES levels in patients with relapsing-remitting multiple sclerosis (RR-MS) treated with cladribine. Acta Neurol Scand. 2004; 109(6):390-392.

17. Liliemark J. The clinical pharmacokinetics of cladribine. Clin Pharmacokinet. 1997;32(2):120-131.

18. Beutler E, Sipe JC, Romine JS, et al. The treatment of chronic progressive multiple sclerosis with cladribine. Proc Natl Acad Sci U SA. 1996; 93(4):1716-1720.

19. Romine JS, Sipe JC, Koziol JA, et al. A double-blind, placebo-controlled, randomized trial of cladribine in relapsing-remitting multiple sclerosis. Proc Assoc Am Physicians. 1999;111:35-44.

20. Ruckemann K, Fairbanks LD, Carrey EA, et al. Leflunomide inhibits pyrimidine de novo synthesis in mitogen-stimulated T-lymphocytes from healthy humans. J Biol Chem. 1998;273:21682-21691.

21. O'Connor PW, Li D, Freedman MS, et al. A Phase II study of the safety and efficacy of teriflunomide in multiple sclerosis with relapses. Neurology. 2006;66(6):894-900. 
22. Confavreux C, O'Connor PW, Fairbanks LD, et al. Safety of teriflunomide in the treatment of multiple sclerosis: results over an 8-year extension. Presented at 26th Congress of the European Committee for Treatment and Research in Multiple Sclerosis (ECTRIMS); October 13-16, 2010; Gothenburg, Sweden.

23. O'Connor PW, Wolinski JS, Confavreux C, et al. TEriflunomide Multiple Sclerosis Oral (TEMSO) Trial Group. A phase III placebo-controlled trial of oral teriflunomide in patients with relapsing forms of multiple sclerosis. Presented at 26th Congress of the European Committee for Treatment and Research in Multiple Sclerosis (ECTRIMS); October 13-16, 2010; Gothenburg, Sweden.

24. Polman C, Barkhof F, Sandberg-Wollheim M, et al. Treatment with laquinimod reduces development of active MRI lesions in relapsing MS. Neurology. 2005;64(6):987-991.

25. Comi G, Pulizzi A, Rovaris M, et al. Effect of laquinimod on MRImonitored disease activity in patients with relapsing-remitting multiple sclerosis: a multicentre, randomised, double-blind, placebo-controlled phase IIb study. Lancet. 2008;371(9630):2085-2092.

26. Comi G, Abramsky O, Arbizu T, et al. Oral laquinimod in patients with relapsing-remitting multiple sclerosis: 9-month double-blind active extension of the multicenter, randomized, double-blind, parallel-group placebo-controlled study. Mult Scler. 2008;14:S37.

27. US Food and Drug Administration. NDA approval letter. January 22, 2010. AMPYRA, NDA no. 022250. http://www.accessdata.fda.gov/drug satfda_docs_label/2010/022250s000REM.pdf. Accessed December 10, 2010.

28. Goodman AD, Brown TR, Krupp LB, et al. Sustained-release oral fampridine in multiple sclerosis: a randomized, double-blind, controlled trial. Lancet. 2009;373(9665):732-738.

29. Goodman AD, Brown TR, Edwards KR, et al. A phase 3 trial of extended release oral dalfampridine in multiple sclerosis. Ann Neurol. 2010;68(4):494-502.

30. Berger T. Current therapeutic recommendations in multiple sclerosis. J Neurol Sci. 2009;287 Suppl 1:S37-S45.
31. The Expanding Treatment Options for Multiple Sclerosis. Proceedings of the 26th Congress of the European Committee for Treatment and Research in Multiple Sclerosis; October 13-16, 2010; Gothenburg, Sweden. Medical Frontiers International; 2010.

32. Counselling Patients on Long-term Disease-modifying Therapy. Counseling Points. 2010;6(1).

33. Gonsette RE. Mitoxantrone immunotherapy in multiple sclerosis. Mult Scler. 1996;1:329-332.

34. Treadaway K, Cutter G, Salter A, et al. Factors that influence adherence with disease-modifying therapy in MS. J Neurol. 2009; 256(4):568-576.

35. Adherence to Disease-Modifying Agents in Relapsing-Remitting Multiple Sclerosis. International Journal of MS Care. 2010;12(2).

36. Turner AP, Williams RM, Sloan AP, et al. Injection anxiety remains a long-term barrier to medication adherence in multiple sclerosis. Rehabil Psychol. 2009;54(1):116-121.

37. Mohr DC, Goodkin DE, Likosky W, et al. Therapeutic expectations of patients with multiple sclerosis upon initiating interferon beta-1b: relationship to adherence to treatment. Mul Scler. 1996;2: 222-226.

38. Portaccio E, Zipoli V, Siracusa G, et al. Long-term adherence to interferon beta therapy in relapsing-remitting multiple sclerosis. Eur Neurol. 2008;59:131-135.

39. Shea SC. Improving Medication Adherence. How to Talk with Patients About Their Medications. Philadelphia, PA: Wolters Kluwer Health, Inc; 2006.

40. Stuve, O. Knowns and unknowns in the future of multiple sclerosis treatment. J Neurol Sci. 2009;287 Suppl 1:S30-S36.

41. Tousignant G, Shaw P. Management of Immunomodulating Therapies in MS Care. Proceedings of the 1st MS Expert Nurse Summit conference; November 20-21, 2010; Vienna, Austria. Montreal, UK, 2010.
Patient Preference and Adherence

\section{Publish your work in this journal}

Patient Preference and Adherence is an international, peer-reviewed, open access journal focusing on the growing importance of patient preference and adherence throughout the therapeutic continuum. Patient satisfaction, acceptability, quality of life, compliance, persistence and their role in developing new therapeutic modalities and compounds to

\section{Dovepress}

optimize clinical outcomes for existing disease states are major areas of interest. This journal has been accepted for indexing on PubMed Central. The manuscript management system is completely online and includes a very quick and fair peer-review system. Visit http://www.dovepress.com/ testimonials.php to read real quotes from published authors. 